\title{
Prospective assessment of the gastroesophageal microbiome in VLBW neonates
}

\author{
Vladana Milisavljevic ${ }^{1,2 *}$, Meena Garg², Ivan Vuletic ${ }^{3}$, Jeff F Miller ${ }^{3}$, Lauren Kim², Tina D Cunningham² \\ and Imke Schröder ${ }^{3}$
}

\begin{abstract}
Background: The distal GI microbiota of hospitalized preterm neonates has been established to be unique from that of healthy full-term infants; the proximal Gl, more specifically gastroesophageal colonization has not been systematically addressed. We prospectively evaluated early colonization of gastroesophageal portion of the Gl tract of VLBW infants.

Methods: This study involved 12 infants admitted to a level III NICU with gestational age (GA) $27+/-0.5$ weeks and birth weight $1105+/-77$ grams. The gastroesophageal microbial flora was evaluated using 16S rDNA analysis of aspirates collected in a sterile manner during the first 28 days of life.

Results: Bacteria were detected in 9 of the 12 neonates. Ureaplasma was the dominant species in the first week of life, however, staphylococci were the predominant bacteria overall. By the fourth week, Gram (-) bacteria increased in abundance to account for $50 \%$ of the total organisms. Firmicutes were present in the majority of the neonates and persisted throughout the 4 weeks comprising nearly half of the sequenced clones. Noticeably, only two distinct species of Staphylococcus epidermidis were found, suggesting acquisition from the environment.

Conclusions: In our neonates, the esophagus and stomach environment changed from being relatively sterile at birth to becoming colonized by a phylogenetically diverse microbiota of low individual complexity. By the fourth week, we found predominance of Firmicutes and Proteobacteria. Bacteria from both phyla (CONS and Gram (-) organisms) are strongly implicated as causes of hospital-acquired infections (HAl). Evaluation of the measures preventing colonization with potentially pathogenic and pathogenic microorganisms from the hospital environment may be warranted and may suggest novel approaches to improving quality in neonatal care.
\end{abstract}

Keywords: Colonization, Gl microbiome, Neonates, $16 \mathrm{~S}$ rDNA

\section{Background}

While transitioning from a relatively sterile intrauterine environment, neonates become colonized with a complex microbial population. Common environmental exposures influencing colonization include maternal vaginal, fecal, or skin microbiota, as well as breast milk or formula feedings, and result in the development of the intestinal microbiome. In contrast to healthy babies, preterm infants have prolonged hospital stays in the neonatal intensive care unit (NICU), and therefore are exposed to

\footnotetext{
* Correspondence: vladana.milisavljevic@cshs.org

'Department of Pediatrics, Cedars-Sinai Medical Center, 8700 Beverly

Boulevard, NT, Suite 4311, Los Angeles, CA 90048, USA

${ }^{2}$ Department of Pediatrics, Neonatal Research Center, David Geffen School of Medicine, University of California Los Angeles, Los Angeles, CA, USA

Full list of author information is available at the end of the article
}

microorganisms specific to the hospital environment. Very low birth weight (VLBW) infants frequently experience hospital-acquired infection (HAI) during prolonged hospitalization, which significantly contributes to their mortality and morbidity. The GI microbiota derived from stool samples of hospitalized, preterm neonates was shown to be different from that of healthy, full-term neonates [1,2]. Examinations of the preterm neonatal fecal microbiota demonstrated a low biodiversity and strong prevalence of Staphylococcus and Pseudomonas species with increasing age of the infant [3-5]. Preterm neonates have an immature immune system, mucous membranes and GI tract; immature intestinal epithelial cells may display exaggerated inflammatory responses to both commensal and pathogenic bacteria [6,7]. In neonates bacteria

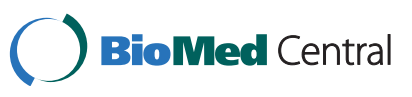


may be more likely to translocate across the GI epithelium to organs and tissues, thereby increasing the risk for systemic infections [8]. The exact mechanisms of such bacterial translocation are still not well understood.

The proximal GI tract can be viewed as a likely "point of entry", however, to date the microbiota of the proximal GI tract in neonates has not been systematically assessed. In adults, colonization of the proximal GI tract by pathogens has been associated with increased incidence of postoperative sepsis [9]. We hypothesized that in the NICU environment opportunistic and pathogenic bacteria, including $S$. epidermidis, influence the formation of the early microbiome of the gastroesophageal portion of proximal GI tract of preterm neonates. The objective of this study was to prospectively investigate the early acquisition of the gastroesophageal microbiome in VLBW neonates using $16 \mathrm{~S}$ rDNA analysis.

\section{Methods}

\section{Patients}

All subjects enrolled in this study were inborn preterm neonates $<32$ weeks gestational age with a birth weight $<1500$ g. They were born and admitted to either the Mattel Children's Hospital UCLA or Santa Monica UCLA Medical Center NICU over a period of six consecutive months (11-1-09 to 4-30-10). Design of both NICUs is a multi-patient, open bay ward. Exclusion criteria were GA $>32$ weeks, birth weight $>1500$ g, lethal congenital malformations or outborn neonates. Neonates with non-lethal congenital anomalies were not excluded. All neonates were enrolled in the study on day 1 of life. Serial proximal GI aspirates were collected for the first 28 days of life and analyzed by week of life. Nasogastric (NG) tube was placed by the bedside nurse under clean conditions after hand washing and appropriate measurements. During the study period, use of gloves was in the guidelines, however, not necessarily always exercised (this has changed since, to the mandatory use of gloves). Correct placement of the NG tube was established by auscultation after injecting 3-5 cc of air. Gastric content has been checked every three hours; if the infant was intermittently feeding, prior to the feeding, if the patient on continuous feedings, every 3 hours during feeding. In some cases the $\mathrm{pH}$ of gastric content was tested to indicate correct placement. For this study purpose, the bedside nurses were instructed to save any gastric aspirate obtained when they checked for residuals. The aspirates were placed on ice and stored in the NICU specimen refrigerator. A member of study team was paged to pick up the samples as soon as collected for further analysis. NG tubes were changed daily. Precautions were exercised that, once collected, samples are handled under sterile conditions. Demographic and clinical data were obtained from review of medical records.

\section{Ethics statement}

The UCLA Institutional Review Board approved the study. Written informed consent was obtained from the parents of all patients shortly after delivery.

\section{Molecular analysis}

The composition of the gastroesophageal microbial flora was surveyed using $16 \mathrm{~S}$ rDNA analysis. Briefly, $1 \mu \mathrm{l}$ of the collected aspirates was directly added to PCR reactions. Control reactions were spiked with E. coli DNA to control for possible acidification of the reaction mix. Universal bacterial 16S rDNA primers 8F (5'-AGAGTTTGATYMT GGCTCAG) and 1510R (5'-TACGGYTACCTTGTTACG ACTT) were used for $16 \mathrm{~S}$ rDNA amplification. The high fidelity, rapid enzyme Phire polymerase (NEB) was used for all amplifications. Amplification reactions were done as follows: $3 \mathrm{~min}$ at $98^{\circ} \mathrm{C}, 25$ cycles of $30 \mathrm{sec}$ at $98^{\circ} \mathrm{C}, 20 \mathrm{sec}$ at $55^{\circ} \mathrm{C}$, and $1 \mathrm{~min}$ at $72^{\circ} \mathrm{C}$, concluded with $5 \mathrm{~min}$ at $72^{\circ} \mathrm{C}$. PCR reactions were limited to 25 cycles to minimize artifacts potentially caused by Phire polymerase. PCR products were agarose gel purified, TOPO cloned into plasmid pCR2.1, and transformed into E. coli TOP10 (Invitrogen). Plasmid inserts were sequenced by Beckman Coulter Genomics. Initially 8 clones were submitted for sequence analysis per positive sample. If the results contained consistently the same type of bacterial species, we assumed that we sequenced the sample to completion. However, if the sequencing results contained several distinct bacterial species, more clones were sent out for sequencing until no new species sequence was obtained (Table 1). If the sequence was $>99 \%$ identical to a bacterial species in the database, we assigned it the name given in the database. All sequences were submitted to the National Center for Biotechnology Information (NCBI) database.

\section{Bacterial isolates}

Collected aspirates were directly plated on tryptic soy agar (Difco) and bacterial colonies were purified by re-

Table 1 Bacterial species and sequenced clones by week per infant with positive aspirates

\begin{tabular}{ccccc}
\hline Infant & Week 1 & Week 2 & Week 3 & Week 2 \\
\hline 1 & 0 & $4(12)$ & $2(8)$ & 0 \\
2 & $5(24)$ & 0 & 0 & 0 \\
3 & $4(12)$ & 0 & 0 & 0 \\
4 & 0 & 0 & $2(8)$ & 0 \\
5 & $2(8)$ & 0 & $2(8)$ & $3(12)$ \\
6 & 0 & $3(12)$ & 0 & 0 \\
7 & 0 & 2 & $2(8)$ & 0 \\
8 & 0 & 2 & $4(12)$ & $3(12)$ \\
9 & $4(12)$ & 0 & $3(12)$ & $6(30)$ \\
\hline
\end{tabular}

The first number indicates the distinct bacterial species that were identified, the number in brackets clones that had been sequenced. 
streaking on the same medium after overnight incubation at $37^{\circ} \mathrm{C}$ under aerobic conditions. Bacterial isolates were identified by $16 \mathrm{~S}$ rDNA sequence analysis and their sequence was submitted to the National Center for Biotechnology Information (NCBI) database.

\section{Data analysis}

The DNA sequences of each clone set were compared to each other to eliminate redundant sequences. Unique sequences were blasted against the non-redundant nucleotide database. Most sequences matched $16 \mathrm{~S}$ rRNA sequences in the database with $\mathrm{E}$ values of 0 and were designated to be the same sequence. For the construction of the phylogenetic tree, sequences were retrieved from the NCBI database. Two 16S rRNA sequences with poor matches to the database were submitted to NCBI. Sequences were aligned using ClustalW, and the phylogenetic tree was constructed with the TREECON software using the Kimura 2 algorithm.

Descriptive statistics were computed for the prenatal and postnatal characteristics from the 12 subjects. The primary outcome of interest was whether or not gastroesophageal aspirates tested positive for bacteria. Since our sample size was very small, Fisher's exact test was used to evaluate the association between clinical variables potentially affecting colonization and the presence or absence of bacteria during the first four weeks of life. Clinical variables evaluated were delivery mode (vaginal or cesarean section), rupture of membranes, chorioamnionitis, type of feedings (formula, breast milk, breast milk plus formula), use of antibiotics, use of famotidine and use of respiratory support. We performed analysis using SAS/STAT software (version 9.2, SAS Institute Inc, Cary, NC).

\section{Results}

\section{Clinical characteristics of the neonatal cohort}

Clinical characteristics, including demographic and clinical data of 12 study subjects are summarized in Tables 2 and 3. Data for 2 of 12 infants were not available after week two of study due to transfer to another facility in one, and a death unrelated to sepsis in the other case.

\section{Statistical analysis}

Analysis of all clinical data using Fisher's exact test showed no association between any of the evaluated clinical variables (delivery mode, rupture of membranes, chorioamnionitis, type of feedings, uses of antibiotics, famotidine and respiratory support) and the presence or bacteria distributed in the four phyla identified in our samples ( $p$-values $>0.05$ ).

\section{Gastroesophageal microbiome evaluated at phyla level} Over four weeks of our study a total of 108 gastroesophageal aspirates were collected from the patient cohort.
Table 2 Clinical characteristics for the 12 subjects enrolled in the study

\begin{tabular}{ll}
\hline Summary of demographic and clinical data & \\
\hline Prenatal characteristics & $\mathbf{n}=\mathbf{1 2}$ infants \\
\hline Gestational age in weeks & $29.2 \pm 0.7^{*}(24-32)$ \\
Birth weight in grams & $1140 \pm 72^{*}(580-1390)$ \\
Male sex & $8(67 \%)$ \\
Cesarean section & $10(83 \%)$ \\
Maternal antenatal steroids & $12(100 \%)$ \\
Maternal antenatal antibiotics & $6(50 \%)$ \\
Maternal chorioamnionitis & $2(17 \%)$ \\
Prolonged rupture of membranes & $4(33 \%)$
\end{tabular}

Postnatal characteristics

RDS requiring intubation/surfactant 9 (75\%)

RDS requiring CPAP - high flow nasal cannula $3(25 \%)$

Umbilical artery or vein catheterization $\quad 11(92 \%)$

PICC line placement $\quad 12(100 \%)$

Antacid use (famotidine) 6 (50\%)

Antibiotics in first $48 \mathrm{hrs}$

Positive blood culture (late onset sepsis)

Positive tracheal culture

$3(25 \%)$

*Mean \pm SEM.

The range of gastroeasohageal aspirates obtained was 4-26 per infant, however, not all the samples obtained from the infant with 26 samples were analyzed, as they were days when the patient had several samples. When there were duplicate samples obtained the same day from an infant, only one was analyzed. Ninety-five samples were analyzed by PCR using universal primers to the $16 \mathrm{~S}$ rDNA gene. Twenty-five cohort samples tested positive for bacteria and

Table 3 Enteral nutrition and clinical variables by week of life

\begin{tabular}{lcccc}
\hline & $\begin{array}{c}\text { Week 1 } \\
(\mathbf{n}=\mathbf{1 2})\end{array}$ & $\begin{array}{c}\text { Week 2 } \\
(\mathbf{n}=\mathbf{1 2})\end{array}$ & $\begin{array}{c}\text { Week 3 } \\
(\mathbf{n}=\mathbf{1 0})\end{array}$ & $\begin{array}{c}\text { Week 4 } \\
(\mathbf{n}=\mathbf{1 0})\end{array}$ \\
\hline Enteral nutrition & 7 & 9 & 9 & 8 \\
Enteral feeds & 2 & 7 & 9 & 7 \\
Breast milk feeds only & 0 & 0 & 0 & 1 \\
Formula feeds only & 5 & 2 & 0 & 0 \\
Breast milk+ formula feeds & 1 & 3 & 4 & 4 \\
Full enteral feedings & & & & \\
Clinical variables & 4 & 5 & 4 & 6 \\
Famotidine & 12 & 2 & 2 & 5 \\
Antibiotics & 12 & 12 & 12 & 12 \\
NG Tube/Repogle & 9 & 4 & 5 & 5 \\
ETT & 3 & 6 & 3 & 1 \\
CPAP/HFNC & 0 & 2 & 2 & 4 \\
Nasal cannula $\mathrm{O}_{2}$ & & & & \\
\hline
\end{tabular}


the PCR products were cloned. A total of 190 clones were sequenced identifying 4 phyla containing 13 bacterial species with 29 subspecies (Figure 1). In 3 of the 12 infants no bacteria were detectable through the 4 weeks of the study (Table 4).

In 9 infants with detected bacteria, Firmicutes constituted the predominant phylum followed by Proteobacteria, Tenericutes and Actinobacteria (Figure 2a). Firmicutes persisted during the course of the 4-week study, comprising approximately $50 \%$ of all clones. Tenericutes, which made up a relatively large population in our samples during the first week of life, markedly diminished and disappeared by week 3 . In contrast, Proteobacteria, constituting a relatively minor population in the first week of life, gradually increased in number over 4 weeks of the study to account for $\sim 50 \%$ of all clones (Figure $2 b$ ).

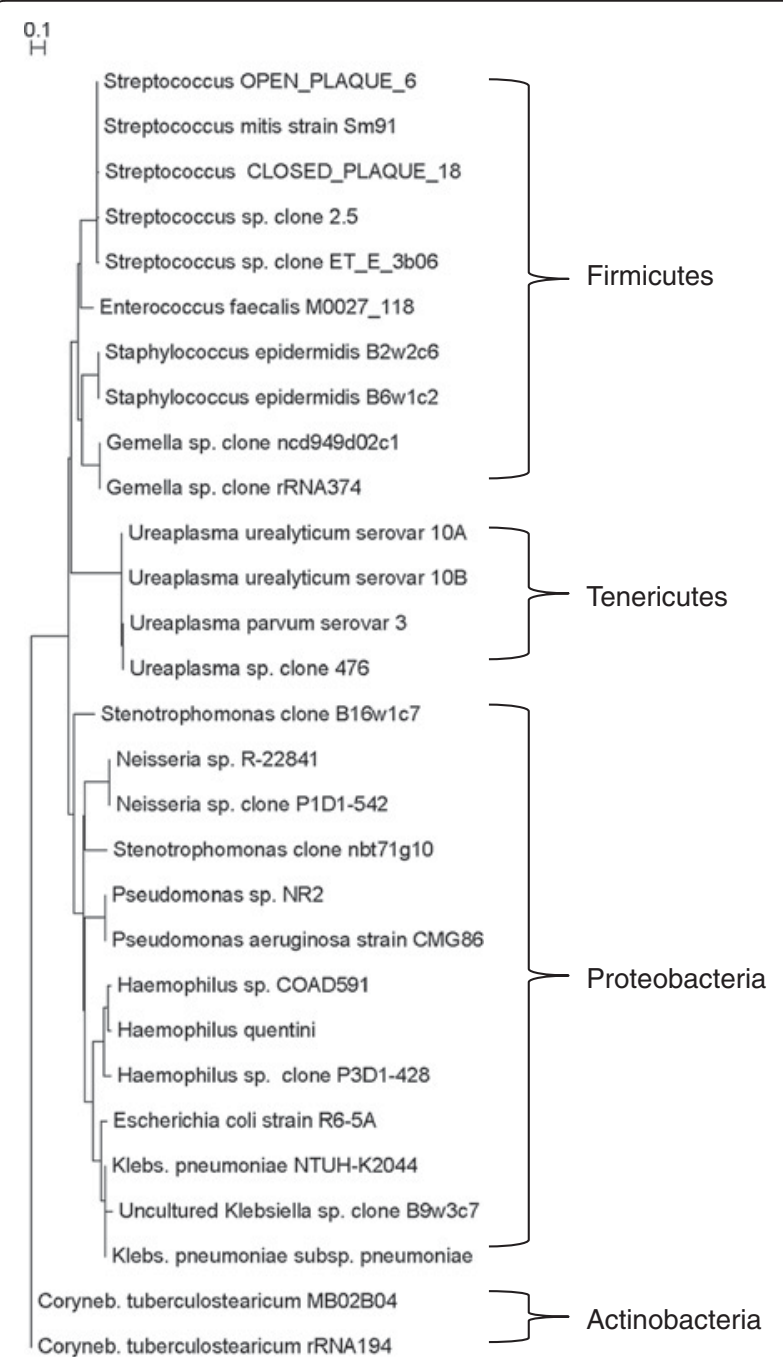

Figure 1 Phylogenetic relationships of bacteria isolated from the gastroesophageal aspirates.
Gastroesophageal microbiome evaluated at genus level A summary of all the bacterial species identified from the gastroesophageal aspirates in 9 of 12 VLBW study subjects is shown in Table 4. Staphylococci were the most commonly detected bacterial species comprising about one third of all sequenced clones (Figure 3). We detected the presence of two reoccurring species of staphylococci, i.e., Staphylococcus epidermidis B2w2c6 (in 6/9 infants positive for bacteria) and S. epidermidis B6w1c2 (in 5/9 infants) (Table 4). We isolated both Staphylococcus species by plating gastric samples directly onto tryptic soy agar and submitted their $16 \mathrm{~S}$ rDNA sequence to the NCBI database.

Streptococcus and Ureaplasma species were the second most abundant bacteria within the sequenced bacterial species library (Figure 2). In contrast to Staphylococcus, the Streptococcus genus consisted of 5 species, all of which were detected in two infants (Table 4 and Figure 1). The Streptococcus species included the alphahemolytic S. mitis Sm91, and streptococci previously identified as part of the oral bacterial microbiome ([10], Parahitiyawa NB, et al, unpublished, submitted 2009 to the INSDC). Gemella, a close relative of Staphylococcus and normally found on human mucous membranes, was detected in week 4 only in one infant.

Ureaplasma species were predominantly present during the first week of life (Table 4, Figure 3). Two Ureaplasma parvum and two Ureaplasma urealyticum serovars were identified in 4 neonates. It is likely that all Ureaplasma bacteria were transmitted during delivery or acquired in utero via infected amniotic fluid [11]. Their marked absence in the later weeks of life suggests that Ureaplasma fails to persist in the gastric environment and/or was eliminated by antibiotic treatment.

Other, less abundant, bacteria detected in the first two weeks of life include Stenotrophomonas, Corynebacterium tuberculostearicum and Enterococcus faecalis species (Figure 4). E. faecalis B6w1c4 was isolated from a gastric aspirate sample by plating on tryptic soy agar.

The overall percentage of Gram (-) organisms including Neisseria, Haemophilus, K. pneumoniae, P. aeruginosa and $E$. coli species increased from $9 \%$ in the week 1 to $50 \%$ of all the organisms in the week 4 (Figures $2 \mathrm{~b}$ and 4 ).

\section{Discussion}

This report illustrates the phylogenetic diversity of the gastroesophageal microbial flora in VLBW infants from birth to 4 weeks of age using $16 \mathrm{~S}$ rDNA analysis of obtained aspirates. All the neonates were enrolled during a period of 6 month. The gastroesophageal portion of the GI tract was chosen for the study since naso/orogastric tubes are placed in all VLBW infants immediately after birth, and may be one of the initial entryways of colonization; stool output is often delayed for up to 5-7 
Table 4 Summary of all the bacterial species identified based on 16S rDNA sequence analysis of upper GI aspirates in the individual VLBW study subjects

\begin{tabular}{|c|c|c|c|c|c|c|c|c|c|c|c|c|c|}
\hline \multirow[b]{2}{*}{ Genus } & \multirow[b]{2}{*}{ Species } & \multicolumn{12}{|c|}{ Infant number } \\
\hline & & 1 & 2 & 3 & 4 & 5 & 6 & 7 & 8 & 9 & 10 & 11 & 12 \\
\hline \multirow[t]{2}{*}{ Staphylococcus } & S. epidermidis B2w2c6** & & $x$ & & $x$ & & & $x$ & $x$ & $x$ & & & $x$ \\
\hline & S. epidermidis B6w1C2** & & & & $x$ & & & $x$ & $x$ & $x$ & & & $x$ \\
\hline \multirow[t]{5}{*}{ Streptococcus } & S. mitis Sm91 & & & & & & & & & & $x$ & & \\
\hline & S. clone ET_E_3b06 & & & & & & & & & & & & $x$ \\
\hline & S. clone 2.5 & & & & & & & & & & & & $x$ \\
\hline & S. Closed Plaque 18 & & & & & & & & & & & & $x$ \\
\hline & S. Open Plaque 6 & & & & & & & & & & $x$ & & \\
\hline \multirow[t]{2}{*}{ Gemella } & G. clone rRNA374 & & & & & & & & & & & & $x$ \\
\hline & G. clone ncd949d02c1 & & & & & & & & & & & & $x$ \\
\hline Enterococcus & E. faecalis B6W1c4** & & & & $x$ & & & & & & & & $x$ \\
\hline \multirow[t]{4}{*}{ Ureaplasma } & U. parvum clone 476 & & $x$ & & $x$ & $x$ & & & & & & & \\
\hline & U. parvum serovar 3 & & & & $x$ & $x$ & & & & & & & \\
\hline & U. urealyticum ser. $10 \mathrm{~A}$ & & & & & & & & & & & & $x$ \\
\hline & U. urealyticum ser. $10 \mathrm{~B}$ & & & & & & & & & & & & $x$ \\
\hline \multirow[t]{3}{*}{ Haemophilus } & H. quentini & & & & & & & & & & $x$ & & \\
\hline & H. COAD591 & & & & & & & & & & $x$ & & \\
\hline & H. P3D1-428 & & & & & & & & & & $x$ & & \\
\hline \multirow[t]{2}{*}{ Stenotrophomonas } & S. clone nbt71g10 & & & & & $x$ & & & $x$ & & & & \\
\hline & S. B16w1c7* & & & & & & & & & & & & $x$ \\
\hline \multirow[t]{3}{*}{ Klebsiella } & K. pneumoniae MGH & & & & & & $x$ & & & & & & \\
\hline & K. pneumoniae NTUH-K2044 & & & & & & $x$ & & & & & & \\
\hline & K. pneumoniae B9w3c7* & & & & & & & & & & $x$ & & \\
\hline \multirow[t]{2}{*}{ Pseudomonas } & P. aeruginosa CMG860 & & $x$ & & & & & & & & & & \\
\hline & P. NR2 & & $x$ & & & & & & & & & & \\
\hline Escherichia & E. coli R6-5A & & & & & & & $x$ & & & & & \\
\hline \multirow[t]{2}{*}{ Neisseria } & N. clone P1D1-542 & & & & & & & & & & & & $x$ \\
\hline & N. R-22841 & & & & & & & & & & & & $x$ \\
\hline \multirow[t]{2}{*}{ Corynebacterium } & C. tuberculostearicum rRNA194 & & $x$ & & $x$ & & & & & & & & \\
\hline & C. tuberculostearicum MB02B04 & & $x$ & & & & & & & & & & \\
\hline
\end{tabular}

*Species were identified in this study; ${ }^{* *}$ bacteria were isolated in pure culture.

days after birth. We demonstrate that in the first 4 weeks of life preterm infants acquire a relatively low complexity bacterial community ranging from 2 to 13 distinct bacterial species (Table 4, Figure 3). This portion of GI tract is not always sterile immediately after birth, as we detected Ureaplasma ssp. and S. epidermidis in the early samples. Maternal Ureaplasma colonization has been associated with preterm labor and chronic lung disease in neonates [12]. In a study by Oue et al. [13], the authors found that detection of Ureaplasma species in gastric fluids was associated with subsequent respiratory colonization, likely promoting the onset of bronchopulmonary dysplasia.

Staphylococci were the predominant organisms during the first four weeks of life in the gastroesophageal microbial flora of infants in our study. This suggests an early and constant exposure of affected infants and/or the capability of Firmicutes to persist despite the use of antibiotics. While 16S rRNA genes sequencing identified two distinct $S$. epidermidis species only, species resolution may require the analysis of additional core genes. However, it is intriguing to consider that populations of the those two S. epidermidis were identified in $50 \%$ of the cohort over the 6 months, implying acquisition from the hospital environment rather than from the mother's skin. S. epidermidis is a normal inhabitant of skin and mucous membranes, however, it is a potential pathogen in patients that are immunocompromised or have indwelling foreign bodies. Since the late 1970s, studies 


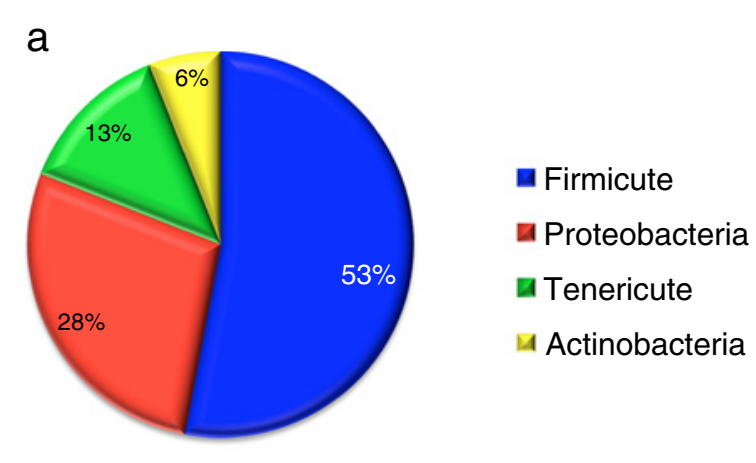

b
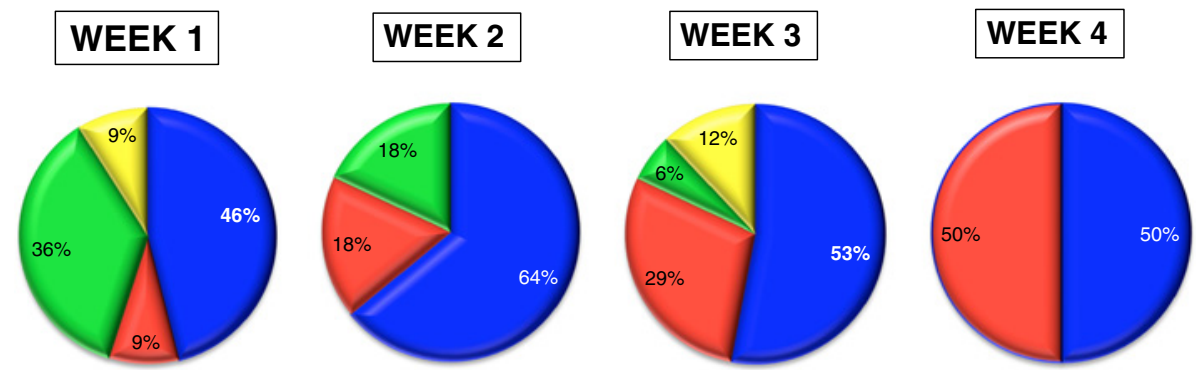

Figure 2 The phylum abundance of bacteria identified in the gastroesophageal aspirates of VLBW neonates. a. Over 4 weeks of study. b. Per week after birth.

have demonstrated that coagulase-negative staphylococci (CONS) are the most common cause of late onset sepsis among NICU low birth weight neonates resulting in 50$70 \%$ of all bloodstream infections [14-16]. In the study by Stoll et al., CONS caused late onset sepsis in $48 \%$ of VLBW infants [17]. Hemolytic peptide $\delta$-toxin, the only identified toxin produced by S. epidermidis, has been associated with necrotizing enterocolitis (NEC), a major morbidity factor in preterm neonates [18]. The pathogenesis of foreign-body-associated infections caused by S. epidermidis is characterized by its ability to colonize polymer surfaces and form biofilms. Bacteria from the

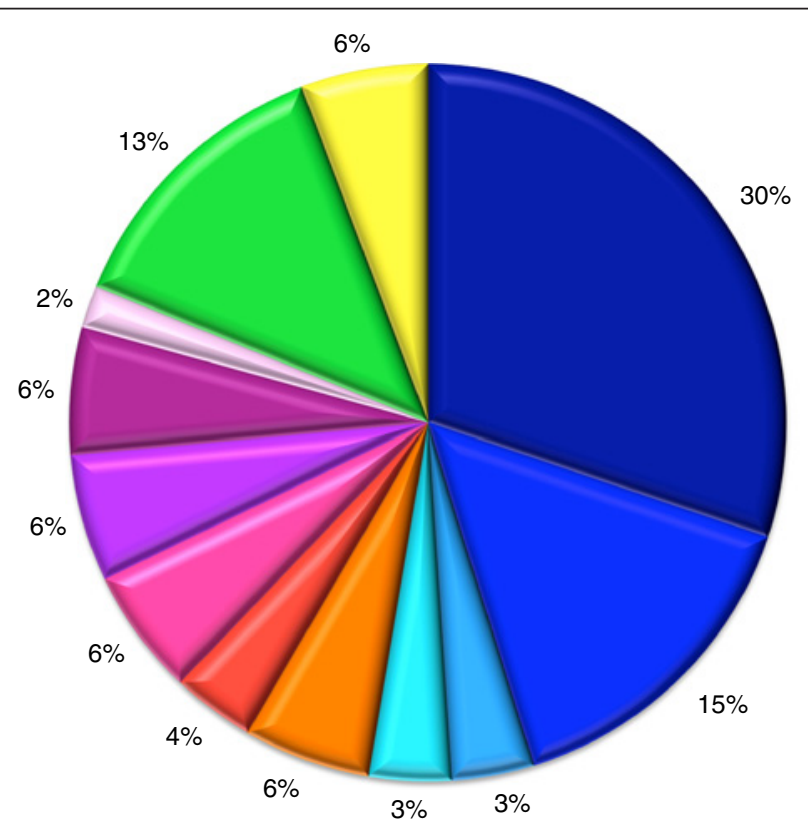

- Staphylococcus

- Streptococcus

a Gemella

Enterococcus

\ Klebsiella

Q Pseudomonas

- Stenotrophomonas

a Haemophilus

a Neisseria

$\checkmark$ Escherichia

a Ureaplasma

$\triangle$ Corynebacterium

Figure 3 The genera diversity of bacteria identified in the gastroesophageal aspirates of VLBW neonates. 


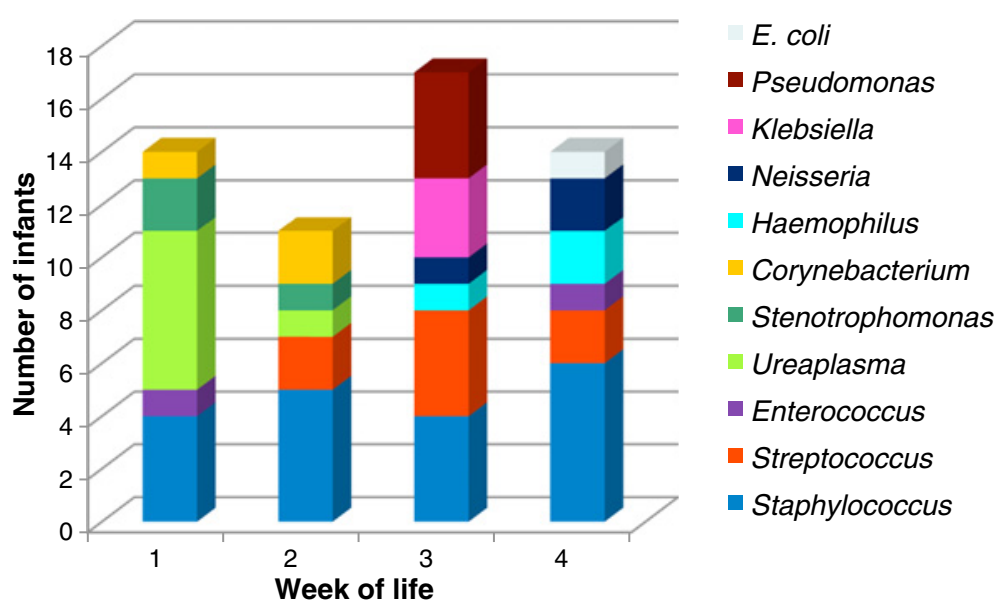

Figure 4 Number of infants colonized by distinct bacteria during the 4 weeks of study.

patient's skin and mucous membranes, or acquired from hands of the hospital staff, can contaminate the polymer during placement of the foreign body or its subsequent care. So far, most studies have focused on intravascular catheters. Hurrell et al. observed biofilm formation inside naso/oro-gastric tubes, and Enterobacteriaceae comprised the major organisms cultured from biofilms $[19,20]$. Mehall et al. isolated S. epidermidis and methicillin-resistant $S$. aureus amongst other bacteria from naso/oro-gastric tubes [21,22]. Naso/orogastric tubes are used to "vent" the stomach, assess gastric residuals and feeding readiness, and for gavage feedings until infant is able to adequately breast/bottle feed. In the case of extremely premature neonates, this can last 10 weeks or longer. Healthcare providers, whose hands are colonized by CONS, still frequently place naso/oro-gastric tubes without using sterile techniques, since there are currently no clear handling guidelines recommended.

Proteobacteria constituted a relatively minor population in the first week of life, but gradually increased in number over the 4 weeks of the study to comprise $\sim 50 \%$ of all clones (Figures $2 \mathrm{~b}$ and 4 ). The increasing abundance of Gram (-) negative bacteria such as Haemophilus, Stenotrophomonas, Klebsiella, Pseudomonas, Escherichia, and Neisseria is particularly concerning since these organisms are frequent pathogens causing HAI [23]. It is likely that these bacteria were acquired from the hospital environment, since they are not known to be present in formula or breast milk feedings. However, we cannot exclude possibility of the initial undetected low-level colonization that increased with time. Infections caused by these organisms are closely associated with their ability to produce biofilms.

We did not find statistically significant relationships between the presence of distinct bacteria and the clinical data collected for each infant. However, our study evaluates a small number of neonates, and the influence of certain clinical practices on bacterial colonization cannot be excluded. In healthy term newborns, the gut microbiota and its evolution over time vary from individual to individual [24]. A twin study that monitored bacterial composition suggests that the initial stages of the infant's GI flora are largely dependent on the specific bacteria to which a baby is exposed [24]. Schwiertz et al. found that preterm infants acquired a low complexity bacterial community after birth, and as a result of hospitalization, developed a similar strain composition over time [1].

Evidence from this and other reports suggests that a shared environment (such as multi patient, open bay ward NICU) can be a major factor. Further studies with an increased cohort size are warranted to evaluate a possible link between the developing GI microbiome and neonatal morbidities. A larger study may support new diagnostic and management approaches in the NICU, as well as suggest interventions for prevention of colonization with potentially pathogenic and pathogenic organisms.

\section{Conclusions}

The gastroesophageal portion of GI tract of VLBW neonates is not always sterile at birth. In the first four weeks of life, VLBW infants managed in the NICU developed a phylogenetically diverse gastroesophageal microbiota of low complexity. In this study, S. epidermidis is overall the predominant organism colonizing gastroesophageal portion of the GI tract throughout the first four weeks of life. By the fourth week of life, the gastroesophageal microbiome of the VLBW infants changed to predominantly compose of Firmicutes and Proteobacteria. Bacteria from both phyla, CONS and Gram (-) organisms, can be biofilm producers and are strongly implicated as causes of hospital-acquired infections (HAI). Our study 
supports the notion that microorganisms present in the hospital environment colonize gastroesophageal portion of the GI tract of preterm neonates, and evaluation of measures that would decrease colonization of immunocompromised patients, such as preterm neonates, with potentially pathogenic and pathogenic bacteria is warranted.

\section{Abbreviations}

16S rDNA: 165 ribosomal DNA; VLBW: Very low birth weight; NICU: Neonatal intensive care unit; PPROM: Preterm premature rupture of membranes; PROM: Premature rupture of membranes; DOL: Day of life;

Gl: Gastrointestinal, RDS, respiratory distress syndrome, LOS, late onset sepsis; CONS: Coagulase-negative staphylococci.

\section{Competing interests}

The authors declare that they have no competing interests.

\section{Authors' contributions}

VM, MG, IM: Substantial contributions to conception and design, acquisition of data, or analysis and interpretation of dat. IV, LK, TDC: Acquisition of data, or analysis and interpretation of data. VM, IM, MG, JFM: Drafting the article or revising it critically for important intellectual content. All authors read and approved the final manuscript.

\section{Acknowledgements}

We would like to thank Dr. Sherin Devaskar for her support, advice, as well as critical review of the manuscript, and Michael Gomez for his technical assistance.

\section{Financial disclosure}

Pediatric Translational Research Group (PTRG) Grant from the Department of Pediatrics, David Geffen School of Medicine at UCLA supported this study (VM). IV was supported by a fellowship from the University of Padua, Italy.

\section{Author details}

'Department of Pediatrics, Cedars-Sinai Medical Center, 8700 Beverly Boulevard, NT, Suite 4311, Los Angeles, CA 90048, USA. ${ }^{2}$ Department of Pediatrics, Neonatal Research Center, David Geffen School of Medicine, University of California Los Angeles, Los Angeles, CA, USA. ${ }^{3}$ Department of Microbiology, Immunology and Molecular Genetics, David Geffen School of Medicine, University of California Los Angeles, Los Angeles, CA, USA.

Received: 17 September 2012 Accepted: 28 February 2013 Published: 5 April 2013

\section{References}

1. Schwiertz A, Gruhl B, Lobnitz M, et al: Development of the intestinal bacterial composition in hospitalized preterm infants in comparison with breast-fed, full-term infants. Pediatr Res 2003, 54(3):393-399.

2. Millar MR, Linton CJ, Cade A, et al: Application of $16 \mathrm{~S}$ rRNA gene PCR to study bowel flora of preterm infants with and without necrotizing enterocolitis. J Clin Microbiol 1996, 34(10):2506-2510.

3. Jacquot A, Neveu D, Aujoulat $F$, et al: Dynamics and clinical evolution of bacterial gut microflora in extremely premature patients. J Pediatr 2011, 158(3):390-396,

4. Morowitz MJ, Denef VJ, Costello EK, et al: Strain-resolved community genomic analysis of gut microbial colonization in a premature infant. Proc Natl Acad Sci U S A 2011, 108(3):1128-1133.

5. Rouge C, Goldenberg $O$, Ferraris $L$, et al: Investigation of the intestinal microbiota in preterm infants using different methods. Anaerobe 2010 16(4):362-370.

6. Levy O, Zarember KA, Roy RM, et al: Selective impairment of TLR-mediated innate immunity in human newborns: neonatal blood plasma reduces monocyte TNF-alpha induction by bacterial lipopeptides, lipopolysaccharide, and imiquimod, but preserves the response to R-848. J Immunol 2004, 173(7):4627-4634.

7. Mshvildadze $\mathrm{M}, \mathrm{Neu} \mathrm{J}$ : The infant intestinal microbiome: friend or foe? Early Hum Dev 2010, 86(Suppl 1):67-71.

8. Duffy LC: Interactions mediating bacterial translocation in the immature intestine. J Nutr 2000, 130:432-6.
9. Reddy BS, Gatt M, Sowdi R, et al: Gastric colonization predisposes to septic morbidity in surgical patients: a prospective study. Nutritition 2008, 24(7-8):632-7.

10. Lu HZ, Weng XH, Zhu B, et al: Major outbreak of toxic shock-like syndrome caused by Streptococcus mitis. J Clin Microbiol 2003, 41(7):3051-3055.

11. Rodriguez N, Fernandez C, Zamora Y, et al: Detection of Ureaplasma urealyticum and Ureaplasma parvum in amniotic fluid: association with pregnancy outcomes. J Matern Fetal Neonatal Med 2010, 24(1):47-50.

12. Milisavljevic V, Cherry J: Mycoplasma and Ureaplasma Infections of the Neonate. In Feigin and Cherry's Textbook of Pediatric Infectious Diseases. 6th edition. Philadelphia, Pennsylvania 19106, USA: Saunders Elsevier; 2009 Volume 75:945-951.

13. Oue S, Hiroi M, Ogawa S, et al: Association of gastric fluid microbes at birth with severe bronchopulmonary dysplasia. Arch Dis Child Fetal Neonatal 2009, 94(1):F17-22. Epub 2008 Aug 1.

14. Freeman J, Epstein MF, Smith NE, et al: Extra hospital stay and antibiotic usage with nosocomial coagulase-negative staphylococcal bacteremia in two neonatal intensive care unit populations. Am J Dis Child 1990, 144(3):324-329.

15. Freeman J, Platt R, Sidebottom DG, et al: Coagulase-negative staphylococcal bacteremia in the changing neonatal intensive care unit population. Is there an epidemic? Jama 1987, 258(18):2548-2552.

16. Gaynes RP, Edwards JR, Jarvis WR, et al: Nosocomial infections among neonates in high-risk nurseries in the United States. National Nosocomial Infections Surveillance System. Pediatrics 1996, 98(3 Pt 1):357-361.

17. Stoll BJ, Hansen N, Fanaroff AA, et al: Late-onset sepsis in very low birth weight neonates: the experience of the NICHD Neonatal Research Network. Pediatrics 2002, 110(2 Pt 1):285-291.

18. Overturf GD, Sherman MP, Scheifele DW, et al: Neonatal necrotizing enterocolitis associated with delta toxin-producing methicillin-resistant Staphylococcus aureus. Pediatr Infect Dis J 1990, 9(2):88-91.

19. Hurrell $E$, Kucerova $E$, Loughlin $M$, et al: Biofilm formation on enteral feeding tubes by Cronobacter sakazakii, Salmonella serovars and other Enterobacteriaceae. Int J Food Microbiol 2009, 136(2):227-231.

20. Hurrell E, Kucerova $E$, Loughlin $M$, et al: Neonatal enteral feeding tubes as loci for colonisation by members of the Enterobacteriaceae. BMC Infect Dis 2009, 9:146.

21. Mehall JR, Kite CA, Gilliam CH, et al: Enteral feeding tubes are a reservoir for nosocomial antibiotic-resistant pathogens. J Pediatr Surg 2002, 37(7):1011-1012.

22. Mehall JR, Kite CA, Saltzman DA, et al: Prospective study of the incidence and complications of bacterial contamination of enteral feeding in neonates. J Pediatr Surg 2002, 37(8):1177-1182.

23. Hidron Al, Edwards JR, Patel J, et al: NHSN annual update: antimicrobialresistant pathogens associated with healthcare-associated infections: annual summary of data reported to the National Healthcare Safety Network at the Centers for Disease Control and Prevention, 2006-2007. Infect Control Hosp Epidemiol 2008, 29(11):996-1011.

24. Palmer C, Bik EM, DiGiulio DB, et al: Development of the human infant intestinal microbiota. PLoS Biol 2007, 5(7):e177.

\section{doi:10.1186/1471-2431-13-49}

Cite this article as: Milisavljevic et al: Prospective assessment of the gastroesophageal microbiome in VLBW neonates. BMC Pediatrics 2013 13:49. 